\title{
Le patrimoine toponymique d'origine et d'influence françaises à l'école en milieu minoritaire : une voie de construction identitaire à emprunter
}

\author{
Carol Léonard \\ Campus Saint-Jean, University of Alberta
}

Résumé

Essentiels à l'orientation et à la localisation, les noms de lieux, ou toponymes, sont des marques identitaires, symboles d'une présence et d'une appropriation du territoire. Ils constituent l'une des composantes majeures du paysage linguistique. Inspiré par une recherche qui a permis d'identifier et de répertorier près de 2500 noms de lieux d'origine et d'influence françaises en Saskatchewan, la présente étude fait le point sur la place accordée à la toponymie dans les écoles francophones en milieux minoritaires. Un constat s'impose. L'étude de la toponymie est quasi absente des programmes d'études. Plusieurs facteurs permettent d'expliquer cet état de fait: la jeunesse de la science toponymique parmi les sciences humaines, et une méconnaissance de la richesse des toponymes d'origine et d'influence françaises dans les provinces et territoires canadiens. Après avoir discuté les obstacles qui se posent à l'identification d'un tel patrimoine et à sa mise en valeur, nous faisons valoir le puissant rôle de paysages linguistiques virtuels que peuvent jouer les cartes toponymiques à l'école.

\section{Abstract}

Place names (or toponyms) are essential to orientation and localization. They are a mark of identity, a symbol of occupation and land appropriation. Place names are among the major elements that constitute the linguistic landscape. Inspired by a research that has permitted to list nearly 2,500 Saskatchewan place names originating from or influenced by the French language in Saskatchewan, the present article reviews the situation regarding the place devoted to the exploration of toponymy in French minority-setting schools. One conclusion jumps out : the study of francophone minorities' toponymy is virtually non-existent. A number of factors help explain this phenomenon : the relatively recent birth of toponymy as a distinct science amongst human sciences, the lack of knowledge regarding the real number of toponyms originating from or influenced by the French language in Canadian provinces and territories. After presenting the numerous barriers to the identification of the toponymic heritage, we call attention to the key role toponymic maps can play as virtual linguistic landscape in the school. 


\section{Introduction}

Il y a de cela quelques années, lors de la fête multiculturelle Mosaic à Regina en Saskatchewan, une carte de belle dimension (10' x 14') de cette province sur laquelle figuraient un peu plus de 1000 points fut exposée dans le pavillon francophone. À côté de chacun des points était inscrit un toponyme français. Cette carte avait été conçue dans le but de faire connaître aux visiteurs du pavillon la richesse de la toponymie d'origine et d'influence françaises de la Saskatchewan. Ma plus grande surprise fut d'entendre certains visiteurs me demander : «Quel pays est représenté sur cette carte? » De toute évidence, la toponymie qui y figurait leur était à ce point étrangère qu'ils ne pouvaient associer ce qu'ils contemplaient avec une province leur étant familière. « C'est la Saskatchewan ! leur répondais-je chaque fois. Le visage médusé des visiteurs était en quelque sorte une invitation à pousser les recherches plus avant, à trouver davantage de toponymes, à viser l'exhaustivité. Depuis cette date, l'inventaire des toponymes d'origine et d'influence françaises en Saskatchewan s'est enrichi. II atteint aujourd'hui près de 2500 . On s'en étonnera peut-être, le toponyme le plus ancien, rivière Danoise (Churchill River), remonte à 1699. Les plus récents, notamment ceux des régions scolaires fransaskoises, datent de quelques années à peine. Dans cette toponymie est inscrite l'histoire de la présence française au cœur des prairies canadiennes. Et pourtant, la toponymie ne figure pas véritablement au programme scolaire du jeune Fransaskois. Son ampleur, sa richesse lui sont inconnues. Qu'en est-il du patrimoine toponymique en milieu minoritaire au Canada? Quelle part joue-t-il dans la construction de l'identité francophone? Tout au long des paragraphes qui suivent, il sera fait état de l'usage des noms de lieux dans un contexte particulier, celui de la salle de classe en milieu francophone minoritaire au Canada. Nous retracerons la récente histoire de cette science qu'est la toponymie et nous situerons la place qu'elle occupe au sein des sciences humaines. Nous ferons aussi état de la place qui lui est réservée en salle de classe et des motifs qui limitent son exploration et son exploitation. Après avoir discuté des obstacles qui se posent à l'identification d'un tel patrimoine, nous aborderons le rôle de premier plan que peut jouer la carte toponymique vue sous l'angle du paysage linguistique.

\section{Médiation cartographique}

L'identité nationale figure en bonne place au nombre des puissants ferments de la cohésion des peuples qui forment un état. L'ethnonyme national (nom d'un peuple) se construit sur la notion de territoire qui en constitue souvent la principale assise. Or, nul ne peut saisir l'étendue du pays ou de la région à l'origine de l'ethnonyme identitaire sans avoir recours à la carte. L'évidence de cette médiation s'impose sitôt que l'on contemple une photographie de la terre prise de l'espace. Le politique presque imperceptible cède sa place au géographique qui domine. Sans parfaite mémorisation des frontières aperçues sur des cartes touristiques ou politiques, il est impossible de reporter sur une photo prise de l'espace les frontières qui délimitent une région, une province, un pays. Sans médiation de la carte, nul ne peut, sauf s'il est insulaire, prendre la mesure de la région qu'il habite. L'utilité de la carte s'affirme chaque jour 
davantage. L'on peut en prendre pour preuve la popularité croissante des systèmes de positionnement géographiques par satellites. La carte est en voie d'occuper plus que jamais une place importante dans nos vies et de se retrouver plus souvent encore au cœur de nos conversations.

\section{Médiation toponymique}

Le nom de lieu ou toponyme doit son apparition à deux fonctions pour lesquelles sa création s'est révélée nécessaire fort tôt dans l'histoire de l'humanité : la localisation et l'orientation. Dès qu'il est énoncé, le nom de lieu acquiert deux autres fonctions. Se chargeant d'histoire, le toponyme devient lui-même site de consignation, un lieu de mémoire (Dorion, 2004). Produit de culture, porté par une langue, il acquiert également une fonction symbolique (Landry et Bourhis, 1997). Élément référentiel fort du paysage linguistique, composant identitaire parmi les plus intimes d'une collectivité linguistique (Lapierre, 2002), le nom de lieu s'affirme chaque jour utile, nécessaire, indispensable aux déplacements comme à leurs évocations.

\section{Pouvoir des noms de lieux}

De tout temps, la carte s'est avérée une représentation conventionnelle avantageuse. On y reproduit la surface terrestre, on y marque les lieux. L'individualité du lieu s'y trouve révélée grâce au point qui en précise la position et au toponyme qui le singularise. Anonyme, le lieu ne trouve pas sa place dans la conversation. Sitôt nommé, il s'humanise et participe aux échanges. Le toponyme médiatise le lieu comme la carte le fait pour la région. Le nom de lieu s'affirme aussi indispensable que la carte. II la complète. La carte devient discours informel (Boudreau, 1989). Elle nous parle.

Mais là ne réside pas l'unique puissance du nom de lieu. Il tire aussi une partie appréciable de son pouvoir du fait que nul ne peut totalement se soustraire à son usage. Ce pouvoir n'est pas uniformément réparti. À l'opposé des noms donnés à des entités éloignées en marge de l'écoumène et qui se rencontrent peu sur les lèvres de la population, les toponymes rencontrés dans le voisinage des lieux habités connaissent en revanche un usage intense. Employés parfois jusqu'à outrance, ils se fondent dans nos habitudes, les imprègnent et finissent par se soustraire à notre attention, à nos sensibilités. Ils se banalisent. Or, le nom de lieu affiche une placidité qui n'est qu'apparente. C'est précisément ses atours, de prime abord inoffensifs, qui offrent au toponyme le couvert à sa puissance et à son action. C'est paradoxalement dans la réification de la charge symbolique du nom, dans son apparente insignifiance, que le nom puise toute sa force. Constamment présent dans les pratiques journalières d'orientation dans l'espace, le toponyme véhicule et légitime une conception du monde, de l'histoire et des rapports sociaux donnant l'illusion d'un complet détachement d'intentions politiques (Azayahu, 1996). II n'apparaît pas créé pour provoquer de vives émotions comme cela est souvent le cas des monuments historiques. Pourtant, le toponyme n'est pas anodin.

Nommer c'est s'approprier (Attali, 1991; Guillorel, 1999; Havard, 2003). Aire de transposition des rêves, des ambitions, des conflits, du bonheur comme du mal de vivre, la toponymie constitue l'une des armatures sur lesquelles s'appuie l'identité de l'individu et celle du groupe. En contexte de contact des langues et d'enjeux linguistiques, le toponyme devient un argument de validation culturelle. De par sa fonction symbolique, le toponyme se retrouve intimement lié au concept de vitalité ethnolinguistique. La prévalence des toponymes dans une langue donnée symbolise non seulement la vitalité linguistique supérieure d'un groupe sur un ou plusieurs autres, mais également la 
place dominante occupée par ce groupe sur l'échiquier social et politique de la région en partage (Bourhis, Giles et Rosenthal, 1981). Subtilement, les toponymes réitèrent jour après jour la réification axiologique d'une certaine interprétation de l'Histoire (Azaryahu, 1996). Lorsque contraint d'utiliser des noms de lieux dans lesquels il ne se reconnaît pas, le parent, par l'usage quotidien qu'il en fait, valide aux yeux de ses propres enfants une toponymie, mais aussi une langue, une histoire et, dans certains cas, une domination. Le message intergénérationnel est passé. Lorsque le nouvel occupant, majoritaire de surcroit, procède à des retranscriptions fautives de toponymes existants, les fixe avec force de loi par l'écrit, les rend intangibles (Breton, 1998), il compromet souvent toute possibilité de restitution d'appellations antérieures. La dénomination scelle la domination.

La toponymie se révèle foisonnante d'informations. Par exemple, une fois identifiée (si ce n'est exhumée), répertoriée et mise en corpus, la toponymie d'origine et d'influence françaises dans les provinces où les francophones sont maintenant en situation de minorité se présente sous les traits d'une riche anthologie dans laquelle se révèlent les manières de dire et de penser, les usages et les connaissances des habitants au cours des âges. La toponymie renseigne tant sur l'étendue des aires habitées que sur les modes d'occupation du territoire. Ainsi, à titre d'exemples, les toponymes Montagne de Tondre (Touchwood Hills) et Montagne de Lime (File Hills) rappellent tous deux la présence en ces lieux de ressources appréciées de la population autochtone soit, d'une part, l'amadou (tondre) utile à l'allumage des feux et, d'autre part, d'une pierre dite « de lime » propre au taillage et au polissage de divers outils. Le découpage synchronique et diachronique de la toponymie fait apparaître les lieux de fréquentation selon les époques et révèle les intentions humaines envers les lieux fréquentés comme à l'égard de ceux qui y habitent. Les calques et les traductions tels que Montagne de Cyprès devenu Cypress Hills ou Sĩsĩp-sākahikan traduit en Lac aux Canards, font plus encore que de renseigner sur les aires de contact entre groupes, ils nous instruisent sur les rapports réels entretenus lors des échanges. La toponymie française de la rivière Churchill au XIXe siècle est en grande partie constituée de toponymes traduits des langues cri et na-déné. Ainsi, le rôle majeur souvent insoupçonné des toponymes et la richesse d'information qu'ils recèlent motivent les interrogations que l'on peut être amené à poser sur la place qui leur est accordée dans les programmes scolaires.

\section{La toponymie, ressource scolaire exploitée?}

II serait surprenant d'entendre un membre d'une communauté minoritaire, si petite soit-elle, affirmer l'insignifiance des noms de lieux issus du groupe auquel il appartient. Au contraire, la préservation et la mise en valeur de ce patrimoine identitaire font souvent partie des aspirations de groupes soucieux de leur identité. La toponymie du Québec nous en fournit un exemple parmi les plus éloquents. Dans cette province où la francophonie affirme sa minorité nord-américaine, la toponymie a fait l'objet d'un traitement particulièrement soutenu. Au cours des décennies marquées par le nouveau réveil de la conscience nationale, le nombre de toponymes officiels en langue française a fait un bond prodigieux. En 1960, on en comptait 45400 (Richard, 2003). En 2006, on en dénombre 181925 (Commission de toponymie du Québec, 2006). La toponymie de cette seule province occupe aujourd'hui près de $40 \%$ de l'ensemble du corpus toponymique officiel de la base de données toponymiques du Canada (figure 1). 


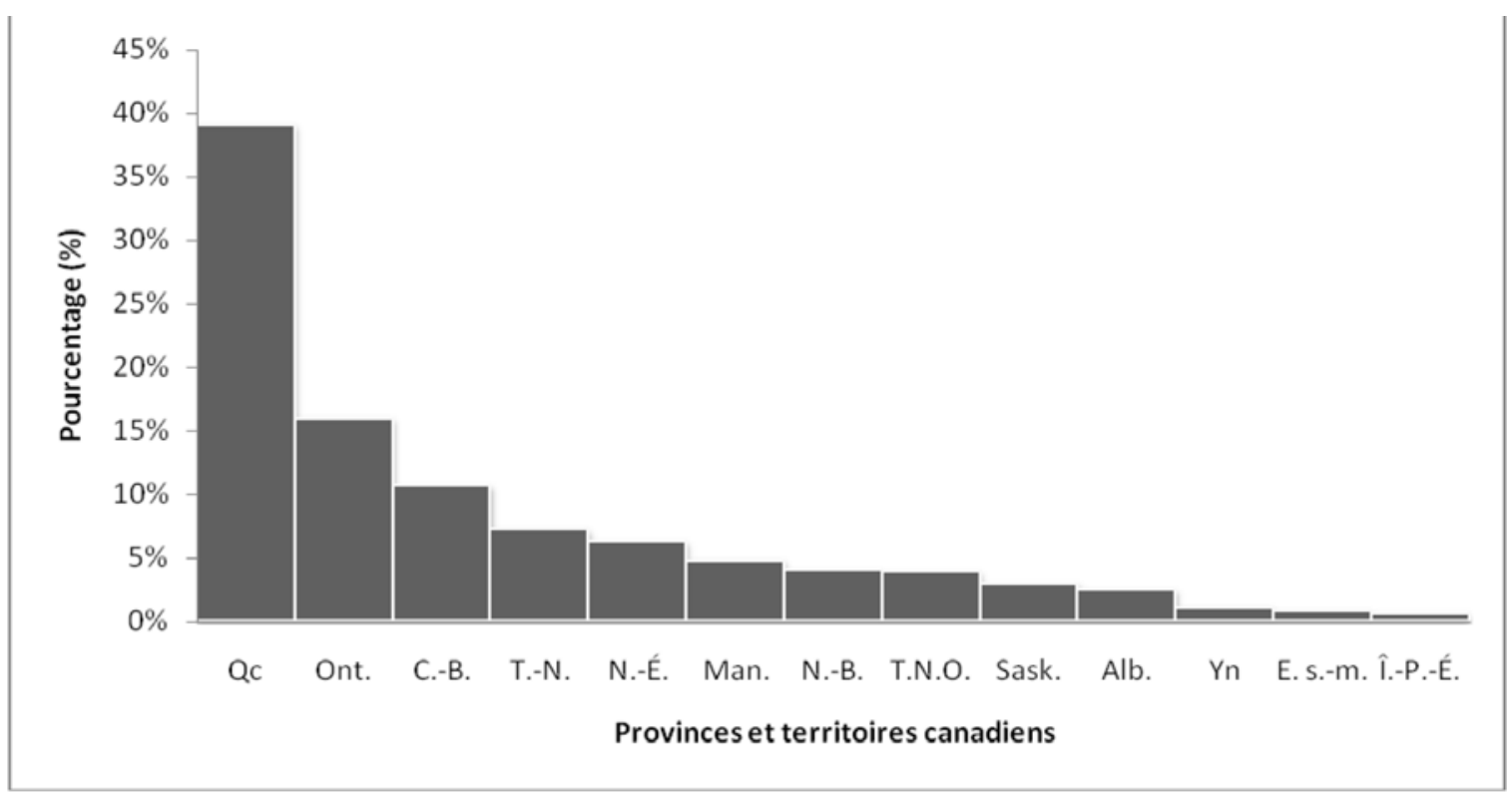

Figure 1: Répartition des entrées de la Banque de données toponymiques du Canada (en \%) selon les provinces, les territoires et les entités sous-marines (Source : Ressources naturelles Canada - données du 17 décembre 2007).

L'attachement à la toponymie en milieu minoritaire au Canada a souvent été rappelé, et ce, depuis fort longtemps. La collision des groupes comme des aspirations qui marquèrent la colonisation de l'Ouest canadien à la fin du XIXe siècle fut l'occasion pour la minorité francophone d'exprimer son attachement à la toponymie française (Frémont, 1922; Prudhomme, 1915). L'intérêt pour la préservation de la toponymie francophone s'est montré aussi vif ailleurs au pays (Lapierre, 1981). II s'est maintenu jusqu'à ce jour. C'est animé d'intentions bienveillantes et soucieuses d'une mise en valeur du patrimoine toponymique acadien que fut organisée à la fin février 2008, une journée de réflexion sur la toponymie et l'odonymie (noms de rues) sous les auspices du Conseil pour l'aménagement du français au Nouveau-Brunswick (CAFNB).

Toutefois, cet intérêt présent et passé pour les noms de lieux, emblèmes de la langue et de la culture, ne semble pas produire d'écho dans les programmes d'études actuels. L'incursion dans le monde de la toponymie d'origine et d'influence françaises semble timide et l'exploration de cette dernière occupe une place modeste dans l'enseignement francophone au Canada. Au Manitoba, en Saskatchewan et en Alberta, les élèves francophones sont bel et bien invités à découvrir la toponymie d'origine française proprement dite alors qu'en Nouvelle-Écosse et en Colombie-Britannique, ce sont les toponymes de toutes origines confondues que les élèves doivent prospecter. On s'en fera une meilleure idée en parcourant le libellé des résultats d'apprentissage dans chacune des provinces :

Manitoba - En deuxième année du programme de français, au cours de l'exploration de la grande communauté canadienne, l'élève francophone aura à se montrer capable d'explorer « différents aspects du Canada, entre autres, les symboles nationaux, l'origine des noms de lieux ainsi que la présence de l'influence de diverses communautés linguistiques et culturelles » (Éducation et Jeunesse Manitoba [EJM], 2003, p. 47). En quatrième année, les résultats se font plus précis. L'élève doit être en mesure de donner des exemples de « l'apport francophone à I'histoire du Manitoba, par exemple l'établissement de Saint-Boniface, les noms de lieux, la langue et la culture, les voyageurs » (EJM, 2003, p. 79). L'élève devra aussi se montrer capable de «relever des exemples de l'apport 
autochtone à l'histoire du Manitoba, par exemple les noms de lieux, l'art, les parcs et les sites historiques, les symboles et les récits, l'assistance aux premiers colons » (EJM, 2003, p. 79).

Saskatchewan- Aux apprenants francophones de quatrième année, la Saskatchewan propose, au programme fransaskois en études sociales, une unité sur l'identité (unité 1) dont le module 2 est intitulé : Les lieux en Saskatchewan (Ministère de l'Éducation de la Saskatchewan [MES], 1997, p. 118). L'élève apprendra en 2.1 (Découvrir les communautés et les gens qui vivent en Saskatchewan) «qu'il existe divers types de communautés dans le nord et le sud de la province ainsi que dans les zones rurales et urbaines » (MES, 1997, p. 120). Ainsi, l'élève découvrira que l'origine des noms «peut être diverse : langues autochtones, nom de la patrie dont on est originaire, nom d'employés du Canadien Pacifique, nom de héros de la Saskatchewan, etc. » (MES, 1997, p. 121). On ajoute à l'alinéa $C$ que l'élève pourra se « familiariser avec les noms des villages et des petites villes de la province » et qu'il faudra laisser « les élèves suggérer des communautés qui, de par leur nom, pourraient être francophones, ou qui auraient pu avoir des habitants d'origine francophone. » On donne pour exemples : Bonne Madonne, Forget, Lisieux, Montmartre, Victoire, Léoville, Saint-Front, Val Marie, Saint-Victor et Laflèche. On ajoute qu'il est approprié d'expliquer "aux élèves que même si le nombre de francophones en Saskatchewan baisse, des familles fransaskoises vivent toujours dans ces communautés » (MES, 1997, p. 121) et on suggère d'amener les élèves à discuter en petits groupes sur les motifs à l'origine du fait que « les Fransaskoises et les Fransaskois de certaines communautés parlent moins français qu'autrefois ». Puis, en 2.2 (Les gens qui se cachent derrière les noms de lieux) (MES, 1997, p. 123) on propose aux élèves l'analyse des lieux de la communauté en songeant aux endroits nommés d'après des noms de personnes, d'en rechercher l'origine en consultant les centres de ressources et même, de recourir à l'entrevue dans la communauté si nécessaire. En plus de réfléchir à l'endroit auquel ils aimeraient donner leur nom, les élèves sont invités à nommer les lieux qu'ils nommeraient d'une autre manière et les noms qu'ils donneraient s'ils étaient autorisés à le faire.

Alberta - En quatrième année, l'un des résultats d'apprentissage du programme d'études sociales mentionne spécifiquement la toponymie. Dans le cadre du résultat d'apprentissage général 4.3 , l'élève francophone doit faire «preuve d'une compréhension et d'un discernement de la manière dont l'Alberta a évolué sur les plans économique, social et culturel depuis 1905 » (Alberta Education, 2006, p. 55). Au résultat d'apprentissage spécifique 4.3.3 l'élève est invité à « explorer d'un œil critique la dynamique sociale et culturelle de l'Alberta en étudiant les questions suivantes et en y réfléchissant ». L'une des huit questions abordées et la seule portant sur la toponymie se lit comme suit : « Comment la toponymie reflète-t-elle les origines des gens qui ont habité, découvert ou développé les lieux ? » (Alberta Education, 2006, p. 56)

Pour mieux aborder cette question, on a mis à la disposition des élèves un «projet d'enquête », sorte de trousse téléchargeable pour faciliter la navigation Internet menant aux ressources toponymiques appropriées. Elle a pour titre : Projet d'enquête : La toponymie et le patrimoine francophone de l'Alberta (LearnAlberta.ca, 2007). On y découvre une fiche à compléter (La toponymie et les communautés francophones) sur laquelle figurent les noms d'une douzaine de communautés franco-albertaines à interpréter.

Nouvelle-Écosse - Au programme d'études de sciences humaines de la quatrième année (résultat d'apprentissage spécifique 3.1), l'élève doit se montrer capable d'identifier «l'origine des peuples fondateurs de sa communauté et ceux de sa province » (Ministère de l'Éducation de la Nouvelle-Écosse, 2006, p. 56). On donne à 
l'élève quelques pistes et, dans l'une d'elles, on prie l'élève « ...d'examiner une carte de la Nouvelle-Écosse en associant des noms de lieux à des peuples fondateurs ».

Colombie-Britannique - Au chapitre des résultats d'apprentissage prescrits du programme d'études en sciences humaines dans le domaine des connaissances en environnement humain, l'on s'attend à ce que les élèves de quatrième année connaissent certains toponymes de la Colombie-Britannique et du Canada. Aucune place particulière n'est accordée à l'étude spécifique des toponymes d'origine et d'influence françaises en ColombieBritannique (Ministère de l'Éducation de la Colombie-Britannique, 2006).

De ce panorama l'on retient aussi l'absence de l'étude et du recours à la toponymie d'origine et d'influence françaises dans les programmes destinés aux francophones des autres provinces et territoires. Ils sont absents en Ontario, alors même qu'elle est la seule province où un répertoire des toponymes provinciaux en langue française a été rédigé à l'intention de la population scolaire francophone (Lapierre, 1981).

Cette présentation sommaire et pancanadienne de la place accordée à la toponymie d'origine et d'influence françaises en salle de classe n'autorise pas un bilan détaillé et une interprétation très nuancée quant aux rapports entretenus par le monde de l'enseignement avec la toponymie. Toutefois, d'une manière générale, là où l'on accorde un peu de temps à l'étude de la toponymie, il s'agit d'un survol rapide. Les noms rencontrés sur les cartes et sur les listes explorées par les élèves sont généralement des toponymes de proximité, des noms de localités plutôt bien connues de la population. Les désignations de lieux habités et non d'entités naturelles (lacs, rivières, baies, îles, montagnes), occupent la première place. Le matériel est présenté en troisième ou quatrième année. II coïncide avec l'aire géographique (territoire et province) généralement étudiée dans chaque province à ces niveaux. À un tel âge, les toponymes doivent être d'un abord aisé et se prêter facilement à une recherche fructueuse. Lorsqu'elle figure aux programmes, l'enquête sur la toponymie d'origine et d'influence françaises demeure non seulement superficielle, mais encore dissoute dans des explorations plus vastes d'analyse de toponymes de toutes provenances linguistiques. Une telle approche ne permet pas d'offrir à la toponymie l'occasion de jouer un rôle dans le processus de construction de l'identité chez les élèves en renforçant les liens qui arriment l'individu à son groupe.

\section{Aux origines de l'absence}

Les constats énoncés ci-haut ne mettent pas en cause le sérieux, le dévouement et la minutie de ceux qui ont charge d'élaborer consciencieusement les éléments des programmes. Les causes sont plus profondes, plus complexes et beaucoup d'entre elles trouvent leur origine dans des contextes extracurriculaires. Elles sont essentiellement de nature historique et cognitive. Elles nous renvoient à la jeunesse de la toponymie en tant que science, à la place qu'elle occupe parmi les sciences humaines et aux difficultés que pose l'exploration du patrimoine toponymique.

\section{La toponymie : science jeune et méconnue}

Bien que l'on se soit intéressé aux noms de lieux depuis la nuit des temps, la toponymie est une science relativement récente. Les deux termes toponymique et toponymie qui la définissent ont fait leur apparition vers 1870 (Baylon et Fabre, 1982). En Amérique, les premières études toponymiques couvrant le domaine français datent du début du XIXe siècle. Le toponymiste Jean Poirier (1972) a répertorié pas moins de 300 articles et une vingtaine de 
livres publiés jusqu'aux années 1970, la plupart de ces contributions sont modestes et caractéristiques d'une discipline qui en est à de premiers tâtonnements. En 1987, la Commission de toponymie du Québec regroupait en une bibliographie pas moins de 1208 titres. En dépit de leur nombre, ces études portent sur des cas particuliers ou des contributions partielles en vue de la création d'inventaires généraux. Plus significatif encore, on remarque le peu de place accordé à l'étude touchant la toponymie de la minorité de langue officielle. Jusqu'à tout récemment, il n'existait aucune étude comparable à celle qu'André Lapierre (1981) a réalisée à l'intention de la population scolaire de l'Ontario. Pour notre part, nous nous sommes livré à un inventaire aussi exhaustif que possible des toponymes d'origine et d'influence françaises en Saskatchewan (Léonard, 2006). Car, enfouis au cœur des inventaires généraux, les toponymes d'origine et d'influence françaises ne sont pas aisément identifiables. Il est difficile de se faire «un portrait » de cette toponymie. Les minorités ont besoin pour elles-mêmes d'inventaires distincts où l'on prend en compte ce qui établit leur spécificité.

\section{La toponymie : science asservie et mésestimée}

La toponymie est une science qui se situe à la confluence de plusieurs disciplines. Certains perçoivent en cela un certain carcan qui, trop longtemps, l'a réduite au rang d'auxiliaire d'autres sciences humaines telles la linguistique, l'histoire et la géographie. Cela s'explique. En Europe occidentale, on a certes connu des guerres terribles et dévastatrices. Elles ont ravagé le sol, mais les toponymes se sont maintenus dans leur vaste majorité. C'est précisément leur pérennité qui les fait reculer à des âges immémoriaux.

La géographie historique n'est pas en reste. Elle a longtemps fait l'étude des noms de lieux en négligeant « une interrogation plus fondamentale sur la nature même de la nomenclature déployée sur la carte, sur les conditions de son inscription, sur ses effets intellectuels et esthétiques, sur les problèmes théoriques sous-jacents à la nomination des lieux » (Jacob, 1992, p. 263). Depuis un peu plus d'une décennie, en divers endroits de la planète, les analyses dans cette veine ont commencé à se succéder (Azaryahu, 1996; Myers, 1996; Parkhurst Ferguson, 1988; Tarkhov, 1992; Yeoh, 1996). Elles rejoignent en cela des études pionnières entreprises au Canada, il y a plus de trente ans, sous la direction des Québécois Henri Dorion et Christian Morissonneau (1972). Ce faisceau de travaux projette les premières lumières sur les enjeux sociopolitiques des toponymies en situation de partage interethnique du territoire.

Ainsi, après avoir été longtemps sous la tutelle d'autres sciences, la toponymie semble de plus en plus pouvoir « parler pour elle-même ». On étudie le nom de lieu pour ce qu'il est, mais aussi, et de mieux en mieux, pour ce qu'il représente.

\section{Le toponyme : objet voisin ou distant}

Un autre motif que l'on doit invoquer pour expliquer le modeste emploi de la toponymie dans les programmes d'études francophones en milieu minoritaire est l'importance des entraves qui se posent à la saisie de ce corpus identitaire. Certaines constituent des obstacles qui peuvent être contournés alors que d'autres court-circuitent l'identification des toponymes et compromettent de manière irréversible leur interprétation et la reconstitution de leur genèse. La toponymie franco-métisse en fournit un exemple (Rivard, 2002). Le départage des origines françaises et amérindiennes de nombre appréciable de toponymes en usage chez les Métis est quasi impossible. 
Parmi les principaux obstacles à la saisie des toponymes, il y a bien entendu les limites de la culture personnelle. La somme d'informations emmagasinées connaît une variabilité infinie d'un individu à un autre. Nul ne peut tout connaître. La langue, la géographie et l'anthroponymie (étude des noms de personnes) sont des domaines complexes. L'influence de la langue et de la culture françaises dans les dénominations Beynes, Lens, Poelcapelle, Illinois, Sioux, Qu'Appelle ou Ponteix n'apparaît pas évidente à chacun. Plus la culture d'un individu est vaste, plus celui-ci pourra capter l'origine ou le sens d'un nom et le replacer dans un contexte social donné. Par exemple, l'étude de la toponymie française en Saskatchewan permet à l'élève de constater la multitude de langues des peuples qui ont formé la France, car nombreux sont les toponymes saskatchewannais qui rappellent les langues que l'on associe à ce pays : Guyader Lake (breton) Strasbourg (alsacien), Lacolle Falls (provençal), Platana ou Cantal (basque).

Au-delà des limites rencontrées chez les individus, il y a les entraves posées par les noms eux-mêmes. Les anthroponymes (noms de personne) sont le siège d'ambigüités linguistiques particulières. Nombreuses sont les entités géographiques et même les voies de communication (rues, boulevards, etc.) dont le toponyme est formé d'un prénom de personne. Bernard, Joseph, Paul, Raymond, Oscar, Maurice sont des prénoms presque banals qui ne laissent pas facilement deviner l'origine linguistique de la personne. Et pour cause. Les noms et les prénoms franchissent aisément les barrières linguistiques. Ils deviennent apatrides. L'identification de l'origine linguistique des personnes à qui les anthroponymes réfèrent n'est pas chose aisée lorsque tous les spécifiques sont accompagnés de génériques dans une seule langue: Oscar Lake, Ernest Lake, Paul Lake, Emma Lake. Lesquels de ces noms évoquent des francophones ? Rien n'est plus difficile à dire. En revanche, si la langue du générique s'accorde à la langue et à la culture de la personne, tout s'éclaire: Oscar Lake, Lac Ernest, Paul Lake, Lac Emma. La langue du générique en situation de contact des langues se révèle un indice de premier ordre souvent indispensable à l'identification du patrimoine toponymique. Les génériques viennent-ils à être imposés dans une seule langue qu'ils indiquent désormais, non seulement le type d'entités géographiques désignées, mais aussi la langue de la majorité occupant le territoire et sa mainmise sur un patrimoine collectif. La présence fortement généralisée de génériques en langue anglaise dans les provinces et territoires où vivent les minorités de langue française au pays représente une entrave de tout premier ordre (Léonard, 2004). À titre d'exemple, le répertoire toponymique de la Saskatchewan contient plus de 12400 toponymes officiels. De ce nombre, environ 5340 toponymes servent à désigner des lacs. Ces toponymes sont tous composés du générique « Lake » à l'exception de 21 d'entre eux formés du générique de langue française, « Lac », ce qui représente moins de $0,4 \%$ de l'ensemble du corpus toponymique provincial.

D'autres entraves sont d'ordre sémantique et résultent d'une graphie fautive. Les erreurs de retranscription qui ont affecté bien des toponymes au cours des âges sont nombreuses surtout lorsqu'elles sont produites sous la main de personnes peu familières avec la langue du toponyme. C'est ainsi qu'un Péchard sera devenu Peackard. Plus volontaire est parfois le refus de reporter les signes diacritiques indispensables à une correcte interprétation. Un Pagé Lake devient Page Lake. II y a aussi les traductions qui dépossèdent les lieux de leur altérité linguistique. Elles sont parfois si discrètes qu'elles ne laissent pas soupçonner leur existence. Ce que la traduction ajoute de clarté sémantique pour la majorité connaît sa contrepartie dans le retranchement de repères identitaires utiles à la minorité.

Pour être perçu, identifié, interrogé, le toponyme doit être à portée de vue et d'ouïe. Les noms disséminés dans les régions éloignées réduisent à presque rien leur impact culturel quotidien. Malheureusement, plus nombreux encore sont les noms de lieux que la distance et les commodités (échelle des cartes inadéquates, voies d'accès inexistantes) rendent inaccessibles. Au Canada, les toponymes d'origine ou d'influence françaises sont dispersés aux 
quatre coins d'un territoire plus vaste que l'Europe occidentale. À titre d'exemple, en Saskatchewan, plus de 1000 kilomètres séparent la région riche en toponymes évoquant la présence des pères Oblats qu'est le nord du lac Athabasca de la région à forte densité de francophones dans le Centre-Sud de la province. Sans une carte toponymique, leur éloignement ne les fait pas participer au processus de formation de l'identité d'une jeunesse qui ne peut l'intégrer à sa représentation des contributions significatives des individus ou du groupe auquel elle s'identifie.

Enfin, il y a les toponymes victimes du temps, disparus dans les oubliettes de l'Histoire. Certains sont perdus à jamais alors que d'autres languissent sur des cartes anciennes dans les centres d'archives ou croupissent entre les pages de manuscrits, de correspondances, de publications anciennes qui attendent d'être ramenés à la lumière du jour.

Mésestimée, distante, enfouie, la toponymie semble opposer une véritable incommodité à son emploi en salle de classe. Le fait est qu'elle est surtout méconnue. Une bonne part du fonds toponymique patrimonial exploitable est laissée en friche. En d'autres termes, si l'on souhaite voir cette ressource utilisée en salle de classe, il faut d'abord qu'un travail soit fait pour la recueillir, l'indexer, en reconstituer le sens et décrire son évolution. II faut l'étaler sur des cartes consacrées à l'identification du patrimoine francophone de manière à ce qu'en voyant chacun des noms de lieux qui y figurent, l'élève, affranchi des servitudes de l'ignorance et de l'équivoque, sache enfin qu'il s'agit là de toponymes d'origine et d'influence françaises, qu'ils sont nombreux et qu'ils témoignent de la pérennité de son groupe sur le territoire, comme de son influence au cours de l'histoire.

\section{L'exemple de la toponymie fransaskoise}

Au terme de notre recherche qui s'est étendue sur une quinzaine d'années, nous avons pu inventorier et détailler plus de 2500 toponymes d'origine et d'influence françaises (Léonard, 2006) (voir l'annexe) dans une province où l'on pourrait s'attendre à en rencontrer peu (carte 1).

Ces quelque 2500 toponymes fransaskois laissent présager ce qu'une recherche visant l'exhaustivité peut permettre de découvrir et d'étaler ailleurs au pays. À la lumière de nos recherches et celles que nous poursuivons en Alberta où près de 1800 toponymes ont pu être identifiés, il est possible d'estimer le fonds dénominatif à environ 8000 , voire même 10000 toponymes d'origine et d'influence françaises dans l'Ouest canadien. En raison de la pérennité de la présence française sur leur territoire, l'Ontario et les provinces maritimes abritent une richesse dénominative dont on peut s'attendre à ce qu'elle soit bien supérieure à celle rencontrée dans l'Ouest.

II n'y a pas que le nombre de toponymes qui soit digne d'intérêt aux yeux d'une jeunesse francophone curieuse de connaître et de reconnaître la marque laissée par son groupe sur le territoire ou dans sa province. Ressource abondante et généreuse, la toponymie se présente comme une porte d'entrée sur un savoir encyclopédique car, en apparence, tout semble avoir servi à nommer : le ciel et l'atmosphère (la Vieille, représentation animiste qui désigne le vent), les minéraux (portage des Épingles), les végétaux (Talle de harts rouges aujourd'hui Willow Bunch, ou la Rivière aux Foins), les animaux (Cabri qui tire son nom de l'antilope), le sol (La Plaine, point ferroviaire), les humains eux-mêmes (Fourche des Gros-Ventres, du nom des amérindiens Atsinas), les usages (Rivière la Puise ou Butte à viande sèche), les coutumes et les croyances (Mission de la Très-Sainte Trinité ). Enfin, on y trouve aussi nombre de transplantations de toponymes français empruntés à d'autres provinces (Candiac [Québec], Huronville [Ontario]) et à d'autres pays (Bazentin et Cantal [France], Belvoir [Angleterre], Belle Plaine [lowa, USA], 
Lucerne [Suisse]). Ces appellations font découvrir l'ailleurs. Le découpage thématique de cette toponymie nous révèle des pratiques dénominatives et des motifs de dénominations qui diffèrent grandement selon les époques : conquérante sous le Régime français, traduite d'une toponymie amérindienne multilingue à l'époque des voyageurs (carte 2), riche en noms de saints avec les missionnaires (carte 3), envahissante lors de la colonisation, dédicatoire après les deux Grandes guerres. La toponymie peut même contribuer à une mise en question des perspectives historiques. Celle de la Saskatchewan suggère un véritable Âge d'or du français dans l'Ouest à l'époque de la traite des fourrures. Les toponymes français si nombreux laissent entendre que sitôt le canot mis à l'eau, c'est en français que l'on descend ou remonte les rivières et que l'on saute ou contourne les rapides. Elle confirme l'adoption par la langue anglaise d'Amérique de nombreux génériques en langue française tels que butte, cache, coteau, coulée, portage, rapide, etc.

Elle nous révèle aussi que des locuteurs anglophones sont à l'origine de très nombreuses appellations en langue française. Citons au passage quelques exemples: Arras S.D., Bapaume S.D., Calais S.D., Cambrai S.D., Courcelette S.D., Festubert S.D., Givenchy S.D., Louvain S.D., Somme S.D., St. Julien S.D., St. Hilaire S.D., St. Quentin S.D., Verdun S.D., Versailles S.D., Vimy Ridge S.D., Ypres S.D. Ces noms d'arrondissements scolaires donnés pour la plupart par des locuteurs de langue anglaise rappellent les sols français et belges baignés du sang de fils tombés aux champs d'honneur pendant la Première Guerre mondiale.

\section{La toponymie - un paysage linguistique virtuel}

L'on ne peut espérer de la toponymie qu'elle livre toute sa richesse si l'exploitation que l'on en fait demeure confinée à l'enseignement des études sociales ou des sciences humaines en troisième ou quatrième année au moment où les jeunes apprenants abordent l'exploration de leur province. Ce serait priver les apprenants d'un savoir et d'un référentiel identitaire majeurs.

Pour livrer son plein potentiel, la toponymie d'origine et d'influence françaises en milieu minoritaire doit être abordée sous l'angle du paysage linguistique et participer au cadre pédagogique global de la formation des apprenants. Des répertoires toponymiques riches d'information doivent être mis à la portée des apprenants et des éducateurs et surtout, des cartes thématiques de belles factures doivent pendre aux murs des classes, des bibliothèques, des agoras et des halls d'entrée des écoles. En un mot, elles doivent être vues pour servir la curiosité des élèves comme celle des parents et des visiteurs. Elles doivent être présentes tout au long de la formation des élèves. Ces cartes doivent devenir des paysages linguistiques permanents, omniprésents, et ce, pour plus d'un motif.

L'existence d'une influence symbolique du paysage linguistique sur la vitalité ethnolinguistique des groupes minoritaires a été clairement établie (Landry et Bourhis, 1997). À cet égard, la prédominance d'une langue dans le paysage linguistique (et plus particulièrement dans le panorama urbain) se présente comme un discours implicite sur la réalité sociolinguistique. II informe sur le poids démographique, le pouvoir de représentation et le statut des différentes communautés linguistiques se partageant un même territoire. La carte géographique est elle aussi un discours. Elle constitue le prolongement virtuel de l'horizon au-delà duquel on ne voit plus. Les noms de lieux qui la constellent sont des constituants du paysage (Berg et Kearns, 2002). À l'image du paysage linguistique, la carte informe et symbolise.

L'exposition de la toponymie d'origine et d'influence françaises ne peut seule faire contrepoids à l'affichage qui constelle le paysage linguistique ambiant. C'est là un autre front sur lequel d'autres actions doivent être menées. 
En revanche, une fois déployées sur des cartes et celles-ci étalées sur les murs en salle de classe, cette toponymie peut participer puissamment à des expériences valorisantes et conscientisantes qui renforceront chez les jeunes apprenants un sentiment de légitimité combien important (Liebkind, Jasinskaja-Lahti et Teräsaho, 2007) et la conscience d'une pérennité de la présence du groupe sur le territoire. Un tel affichage peut-il avoir un " effet d'entraînement » sur l'usage de la langue du groupe minoritaire ? Peut-il concourir à la mobilisation des jeunes et à une expression plus affirmée de leur identité ? Dans quelle mesure l'intégration du savoir toponymique chez les apprenants peut-elle avoir un effet sur la vitalité linguistique de leur groupe? II y a là place à des hypothèses qu'il conviendra de vérifier sitôt une mise en pratique soutenue et acquise de l'exposition des jeunes francophones minoritaires à ce patrimoine linguistique, historique et géographique qu'est la toponymie. D'ici là, un travail doit être entrepris afin de rendre ce patrimoine accessible, tant sous forme de répertoires que de cartes.

\section{Références}

Alberta Education (2006). Études sociales : de la maternelle à la douzième année, Quatrième année. L'Alberta : terre, histoires et récits. Études sociales. Document téléaccessible à l'adresse URL <http://education.alberta.ca/media/606345/quatrieme.pdf > (consulté le 2 mars 2008).

Attali, J. (1991). 1492. Paris : Fayard.

Azaryahu, M. (1996). The power of commemorative street names. Environment and Planning D: Society and Space, 14, 311-330.

Backhaus, P. (2007). Linguistic landscapes : A comparative study of urban multilingualism in Tokyo. Clevedon : Multilingual Matters.

Baylon, C. et Fabre, P. (1982). Les noms de lieux et de personnes. Paris : Nathan.

Berg, L. D. et Kearns, R. A. (1996). Naming as norming: 'race', gender, and the identity politics of naming places. Aotearoa/New Zealand. Environment and Planning D: Society and Space, 14(1), 99-122.

Berg, L. D. et Kearns, R. A. (2002). Proclaiming place : Towards a geography of place name pronunciation. Social \& Cultural Geography, 3(3), 283-302.

Boudreau, C. (1989). L'analyse de la carte ancienne: quand la carte sert à autre chose qu'à localiser. Stage international de formation en toponymie / Stage de formation en gestion toponymique. Acte du stage tenu à Québec du 7 au 19 août 1988 et organisé par la Commission de toponymie du Québec (Vol. 13), (p. 323-333). Québec : Gouvernement du Québec, Commission de toponymie.

Bourhis, R. Y., Giles, H. et Rosenthal, D. (1981). Notes on the construction of a "Subjective vitality questionnaire" for ethnolinguistic groups. Journal of Multilingual and Multicultural Development, 2, 145-155.

Breton, R. (1998). La restitution des toponymes en Inde. In S. Wydmusch (Éd.), La Toponymie, un patrimoine à préserver : en Inde, en Gascogne, en Alsace, vers une politique de réhabilitation des anciens toponymes? (p. 15-31). Paris : L'Harmattan.

Cohen, S. B. et Kliot, N. (1992). Place-names in Israel's Ideological Struggle over the Administered Territories. Annals of the Association of American Geographers, 82(4), 653 -680.

Commission de toponymie du Québec. (2006). Rapport annuel de Gestion 2005-2006. Québec : Gouvernement du Québec.

Dorion, H. (1993). À qui appartient le nom de lieux? Onomastica Canadiana, 75(1), 1-10.

Dorion, H. (1994). La toponymie : un patrimoine menacé ? Langue nationale et mondialisation : enjeux et défis pour le français (p. 349-357). Québec, Actes du Séminaire des 25, 26 et 27 octobre 1994.

Dorion, H. (2004). Toponymie et enseignement : quelques réflexions pratiques. Onoma 39, 99-108.

Dorion, H. et Morissonneau, C. (1972). Les noms de lieux et le contact des langues - Place names and language contact. Québec : Presses de l'Université Laval.

Éducation et Jeunesse Manitoba. (2003). Sciences humaines Maternelle à 8 - Programme d'études : Cadre manitobain des résultats d'apprentissage. Winnipeg : Ministère de l'Éducation et de la Jeunesse du Manitoba.

Frémont, D. (1922). Des noms français pour nos centres français. Bulletin de la Société de géographie de Québec, 16(4), $222-224$.

Gorter, D. E. (2006). Linguistic Landscape: A new Approach to Multilingualism. Clevedon : Multilingual Matters.

Guillorel, H. (1999). Toponymie et politique. In S. Akin (Éd.), Noms et re-noms : la dénomination des personnes, des populations, des langues et des territoires (p. 61-91). Rouen : Université de Rouen.

Havard, G. (2003). Empire et métissages : Indiens et Français dans le Pays d'en Haut, 1660-1715. Sillery, Québec: Septentrion. Jacob, C. (1992). L'empire des cartes : approche théorique de la cartographie à travers l'histoire. Paris : A. Michel. 
Landry, R. et Bourhis, R. (1997). Linguistic landscape and ethnolinguistic vitality : an empirical study. Journal of Language and Social Psychology, 16, 23-49.

Lapierre, A. (1981). Toponymie française en Ontario. Montréal : Éditions Études vivantes.

Lapierre, A. (1990). Prolégomènes à l'onomastique au Canada français. In J.-C. Boulanger (Éd.), Actes du XVIe Congrès international des sciences onomastiques, Université Laval 16-22 août 1987 (p. 365-373). Québec : Presses de l'Université Laval.

Lapierre, A. (2002). Le toponyme comme marqueur identitaire en Amérique française. In P. Lanthier et P. Savard (Dir.), Constructions identitaires et pratiques sociales : actes du colloque en hommage à Pierre Savard tenu à l'Université d'Ottawa les 4, 5, 6 octobre 2000. Ottawa : Presses de l'Université d'Ottawa.

LearnAlberta.ca (2007) Projet d'enquête : La toponymie et le patrimoine francophone de l'Alberta (4.2.2/4.3.3) Document téléaccessible à l'adresse URL <http://www.onlineguide.learnalberta.ca/contentog/espdquat/html/toponymieetpatrimoine_pde_links.html> (Consulté le 3 septembre 2008).

Léonard, C. J. (2004). Une toponymie voilée : effets de l'unilinguisme des génériques sur la toponymie fransaskoise. Dans S. Langlois et J. Létourneau (Dir.), Aspects de la nouvelle francophonie canadienne (p. 271-292). Québec : Presses de I'Université Laval.

Léonard, C. J. (2006). Une toponymie voilée : problématique des noms de lieux particulière à une minorité canadienne, la Fransaskoisie. Thèse de doctorat, Université Laval.

Liebkind, K., Jasinskaja-Lahti, I. et Teräsaho, M. (2007). Ingroup vitality and intergroup attitudes in a linguistic minority. Scandinavian Journal of Psychology, 48(5), 409-418.

Myers, G. A. (1996). Naming and placing the other : power and the urban landscape in Zanzibar. Tijdschrift voor Economische en Sociale Geografie, 87, 237-246.

Nicolaisen, W. F. H. (1990). Placenames and Politics. Names, 38(3), 193-207.

Ministère de l'Éducation de la Colombie-Britannique. (2006). Sciences humaines M à 7 Version partielle (2006) du programme d'études contenant les sections suivantes : Résultats d'apprentissage prescrits - Rendement de l'élève (Indicateurs de réussite) - Ressources d'apprentissage (Introduction). Vancouver : Ministère de l'Éducation.

Ministère de l'Éducation de la Nouvelle-Écosse, Direction des services acadiens et de langue française (2006). Programme d'études - Sciences humaines, $4 e$ à $6 e$ année. Document téléaccessible à l'adresse URL <http://csap.ednet.ns.ca/cours/sciences\%20humaines\%204\%20a\%206\%20-\%202006.pdf> (consulté le 1er mars 2008).

Ministère de l'Éducation de la Saskatchewan (1997). Programmes fransaskois : Sciences humaines : Programme d'études de la $4^{e}$ année. Guide d'activités. Regina : Ministère de l'Éducation.

Parkhurst Ferguson, P. (1988). Reading City Streets. French Review, 61, 386-397.

Poirier, J. (1972). Aperçu général sur la toponymie canadienne-française. Onoma, XVII, 262-266.

Prudhomme, L. A. (1915). Noms historiques de la langue Française au Nord-Ouest canadien. Bulletin de la Société de géographie de Québec, 9, 4:195-209, 195:283-293, 196:348-364.

Richard, M. (2003). La commission de toponymie du Québec, parcours 1912-2002 : neuf décennies de gestion publique des noms de lieux. Revue d'aménagement linguistique, 167-182.

Rivard, É. (2002) Territorialité métisse et cartographie du Nord-Ouest canadien au XIXe siècle : exploration cartographique et toponymique, Cahiers franco-canadiens de l'Ouest, 14 (1-2), 7-32.

Tarkhov, S. A. (1992). From Karlo-Libknekhtovsk and New York to Propoysk and Rastyapino? : How Place Names Are Changing in the Former USSR. Post-Soviet Geography, 33, 454-462.

Yeoh, B. S. A. (1996). Contesting space : power relations and the urban built environment in colonial Singapore. New York : Oxford University Press. 


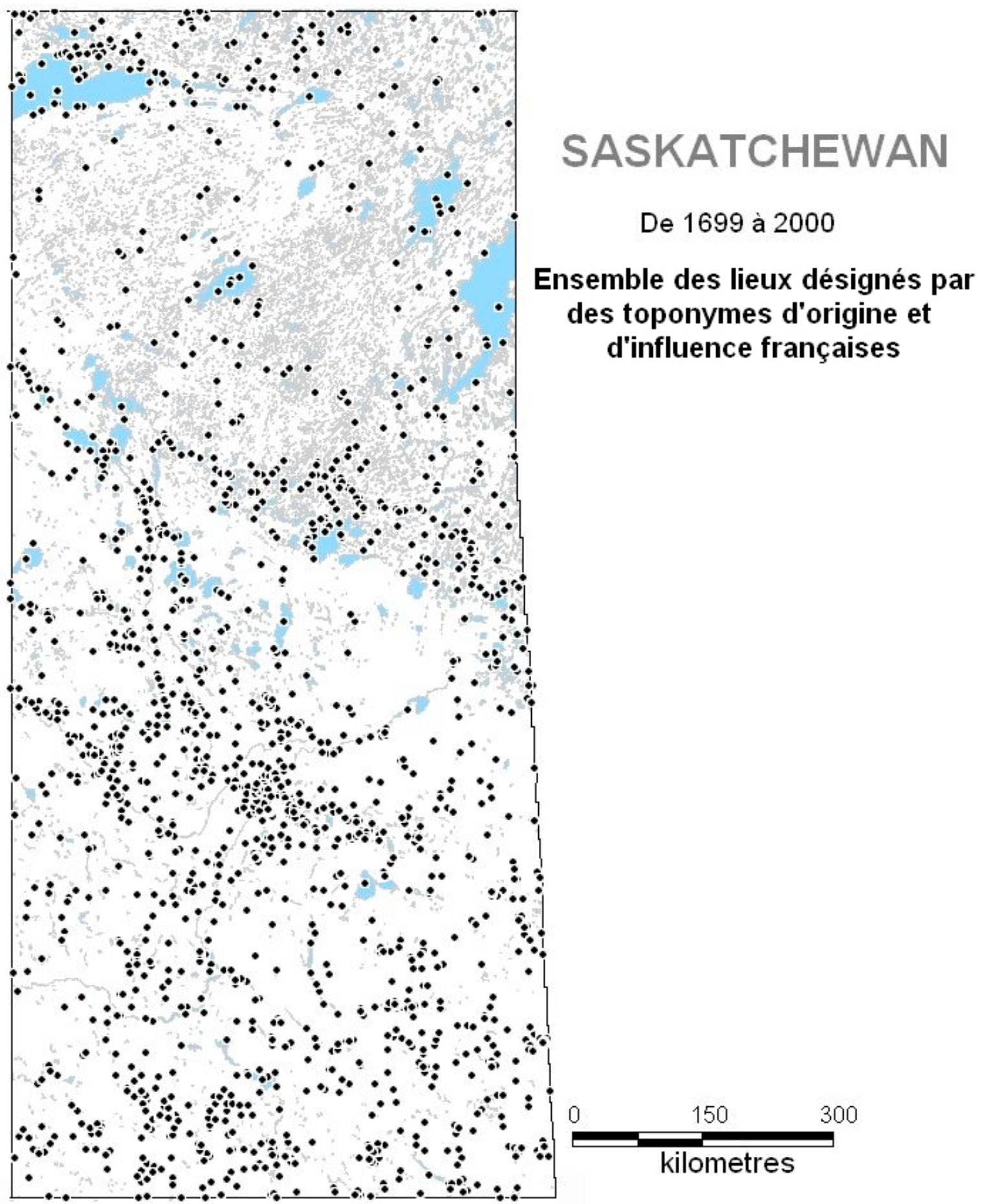

Source : Léonard, C. J., 2006. 


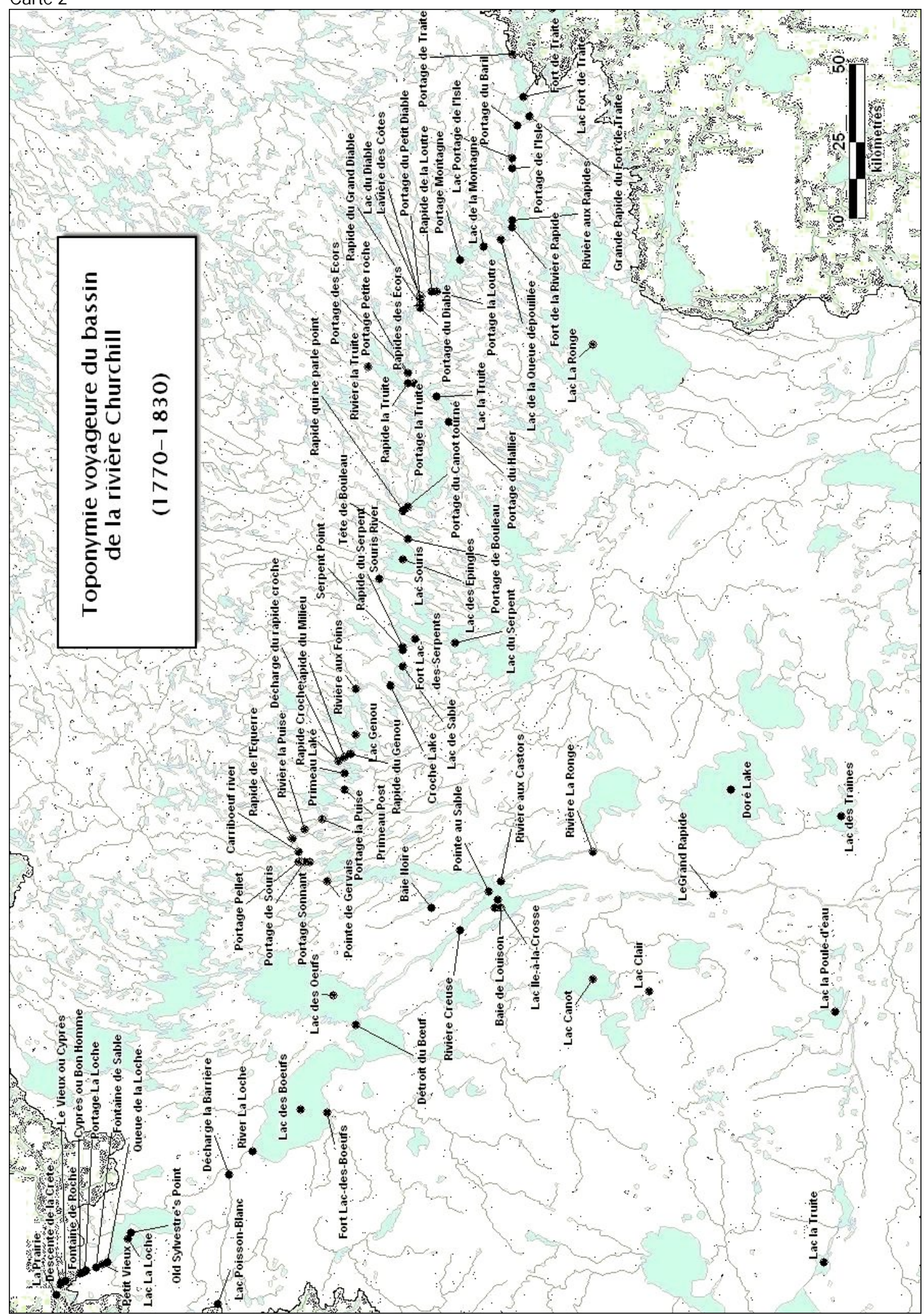

Source : Léonard, C. J., 2006. 

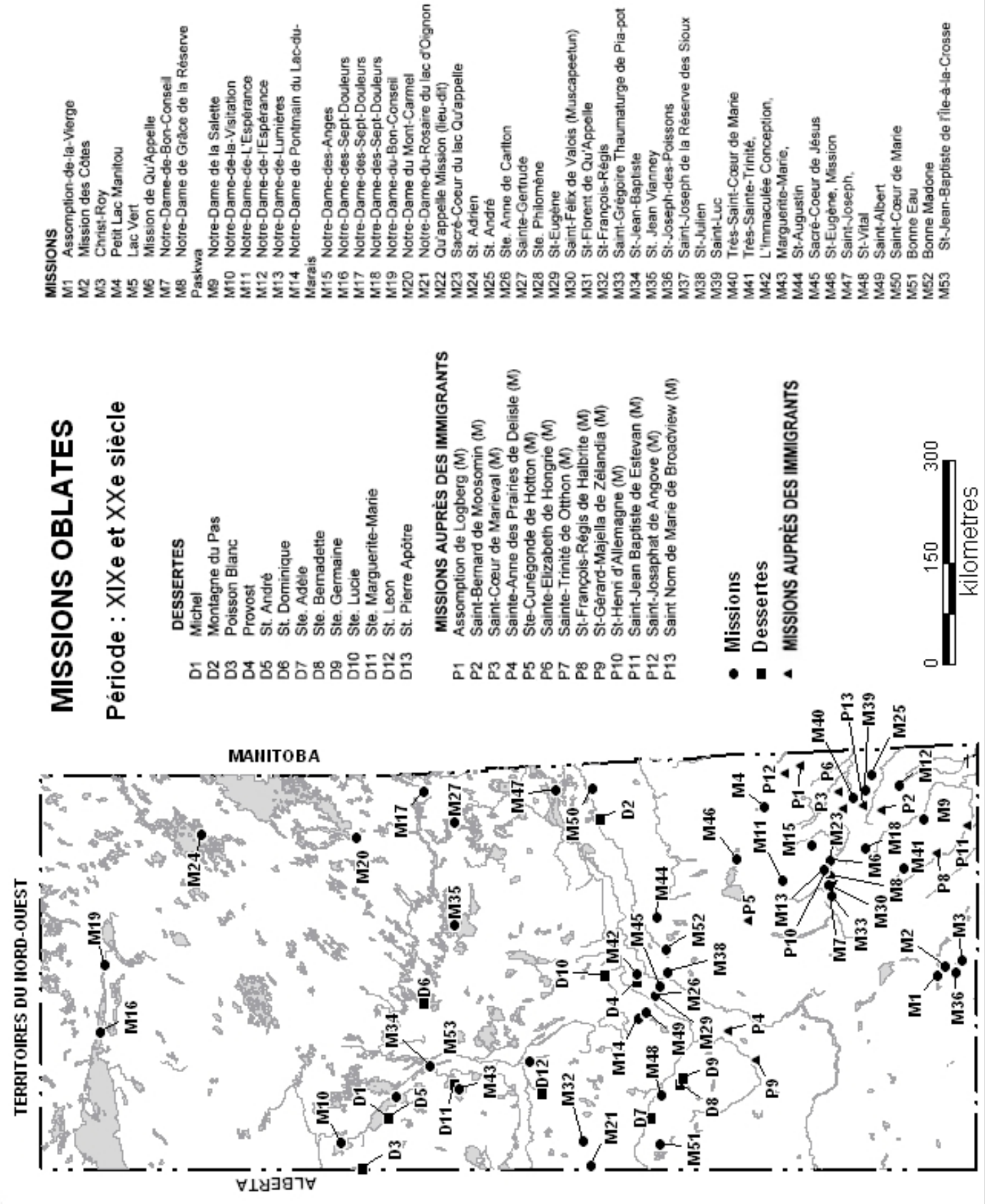

Source : Léonard, C. J., 2006. 


\section{Appendice}

Aux fins d'illustration, voici certains toponymes tirés de notre inventaire des noms de lieux d'origine et d'influence françaises de la Saskatchewan. En raison de l'ampleur de la nomenclature, ne sont présentés ici que les toponymes dont les termes spécifiques (noms propres) commencent par la lettre « $A$ » et « $B$ ». On notera quelques particularités, notamment l'absence des signes diacritiques (accents) appropriés chez certains de ces toponymes. C'est dans cet état que plusieurs d'entre eux ont été officialisés par l'autorité toponymique canadienne. Voici enfin le détail de quelques abréviations employées: «S.D.» School District, «R.C.S.D.» Roman Catholic School District, «R.C.P.S.D » Roman Catholic Public School District et « R.M. » Rural Municipality.

\begin{tabular}{|c|c|c|}
\hline A la Corne & Arras S.D. N 3969 & Batoche \\
\hline A la Corne Rapids & Arsenault Lake. & Batoche Ferry \\
\hline Abraham Bay & Arsenault River & Batoche Lake \\
\hline Adelard's Lake & Assiniboia-Gravelbourg & Batoche Provincial \\
\hline Aigle, Montagne d' & Assiniboilles, Rivière des & Constituency \\
\hline Aimee Lake & Assiniboine, Lac & Batoche Territorial \\
\hline Air Ronge & Assomption de Logberg & Constituency \\
\hline Aisne S.D. N 3908 & Assomption-de-la-Vierge & Batoche, Lieu historique \\
\hline Albert Lake & Athabaskaw, Petite rivière & national de \\
\hline Albertown & Aubiche, Rivière & Batoche's Fort \\
\hline Albertsville S.D. $N^{\circ} 3420$ & Aubichon Arm & Bauval Voir : Beauval \\
\hline Albertville & Aubichon Lake & Bayard S.D. N 4159 \\
\hline Albertville S.D. N 3420 & Aubretia & Bazentin \\
\hline Alberville & Audubon Park & Beatrice Lake \\
\hline Aldina & Augier Lake & Beau Bay \\
\hline Aldina S.D. $N^{\circ} 4760$ & Auriat Island & Beauchamp \\
\hline Alma & Aurice, Portage de & Beauchamp S.D. Nº 4141 \\
\hline Alma S.D. N 505 & Aussant S.D. $N^{\circ} 2373$ & Beaudreau Lake \\
\hline Alwinsal & Auvergne R.M. $N^{\circ} 76$ & Beaudry Lake \\
\hline Amadou, Collines d' & Auvergne-Wise Creek & Beaufield \\
\hline Amiens & Pasture & Beaufield Lake \\
\hline Amiens Junction & Avoine, Rivière d' & Beaufield S.D. № 3169 \\
\hline Amiens Railway & Babiche Lake & Beaulieu Lake \\
\hline Subdivision & Bachelu Lake & Beau-Poisson, Lac \\
\hline Amyot Lake & Baies d'ours, Falaise des & Beaupré Creek \\
\hline Amyot Lake & Baies, île aux & Beaupré Creek \\
\hline Andree Lake & Balleau's House & Recreation Site \\
\hline Aney Lake & Bapaume & Beaupré Lake \\
\hline Anglais, Chenal des & Bapaume Park S.D. N & Beausite S.D. N 3721 \\
\hline Anglais, Lac des & 3877 & Beauval \\
\hline Anglais, Petite rivière aux & Bapaume Pasture & Beauval Forks \\
\hline Anglais, Rivière aux & Bapaume S.D. N 4135 & Beauvalon S.D. N 5156 \\
\hline Anglais, Rivière des & Baptiste Island & Bechard \\
\hline Anglo-Rouyn Mine Site & Baptiste Lake & Bedard Creek \\
\hline Anne Lake & Baptiste Lake & Bedard Creek S.D. N ${ }^{\circ}$ \\
\hline Annette & Baptiste Lake & 4904 \\
\hline Annette Lake & Baribeau Lake & Bedard Island \\
\hline Anse au Poisson, Coulée & Baril, Portage du & Begin Pasture \\
\hline de l' & Barrière, Décharge la & Begin S.D. $N^{\circ} 3346$ \\
\hline Antoine Lake & Barrière, La & Begrand Lake \\
\hline Antoine Point & Barrière, La & Belanger \\
\hline Arc, Rivière d' & Barrière, La rivière & Bélanger \\
\hline Arcand Island & Barrière, Portage la & Belanger Creek \\
\hline Argonne S.D. Nº 2495 & Barrot Lake & Belanger Island \\
\hline Arleux & Bassin, Lac & Belanger Lake \\
\hline Armentieres S.D. $N^{\circ} 4821$ & Bataille, Rivière à & Belanger Point \\
\hline Armorin & Bâtards, Butte des & Belanger R.C.S.D. N ${ }^{\circ} 25$ \\
\hline
\end{tabular}

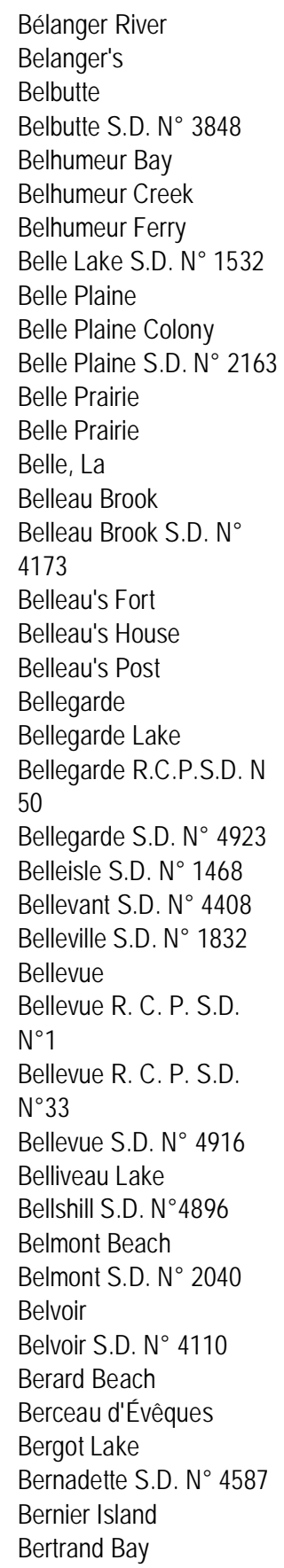




\begin{tabular}{|c|c|c|}
\hline Berube Game Lake & Bois, Montagne de & Bourgogne \\
\hline Preserve & Bois, Petite montagne de & Bousquet Lake \\
\hline Bérubé Lake & Bois, Portage des & Bout du Bois \\
\hline Berube S.D. N 3051 & Boissiere & Boutin \\
\hline Besnard Creek & Boissiere Lake & Boutin's Butte \\
\hline Besnard Lake & Bon Accord S.D. N 4237 & Bouvier \\
\hline Besnard Lake Prov. & Bon Dieu de Jos Gray & Bouvier Creek \\
\hline Recreation Park & Bon Homme & Bouvier Lake \\
\hline Besse Lakes & Bon Secours S.D. N 1489 & Bouvierville \\
\hline Bessette Lake & Bon Seigneur Lake & Boyer Lake \\
\hline Betbeder Coulee & Bonne Eau & Boyer Lake S.D. N 4331 \\
\hline Bethléem du Nord & Bonne Eau, Mission & Bras Cassé, Rivière \\
\hline Beynes & Bonne Madone & Bras, Rivière du \\
\hline Beynes Pasture & Bonne Madone Beach & Breland Lake \\
\hline Beynes S.D. Nº 815 & Bonne Madone Lake & Bremner Lake \\
\hline Biche, Fort la & Bonne Madone, Mission & Bremner S.D. Nº 2921 \\
\hline Biche, Montagne à la & Bonne Madonne S.D. N & Breynat Lake \\
\hline Biche, Petite montagne de & 1343 & Briere Lake \\
\hline la & Bonne Plaine (loc.) & Briese Lake \\
\hline Biche, Rivière à la & Bonneau Lake & Brochet Bay \\
\hline Biche, Rivière la & Bonneauville & Brochet Isle \\
\hline Biche, Rivière la & Bonneville Lake & Brochet, lac de \\
\hline Biches, Rivière aux & Bonsecours & Brochet, Rivière \\
\hline Bienfait & Bonville Lake & Brochu Lake \\
\hline Bienfait S.D. $N^{\circ} 277$ & Bonvouloir & Brûlé Lake \\
\hline Bienfait S.D. N 897 & Bonvouloir S.D. N 3260 & Brunelle Lake \\
\hline Bienfait Town S.D. $N^{\circ}$ & Bosse, Ruisseau de la & Buffalo Coulée \\
\hline 1787 & Bouchard Lake & Butor, Lac \\
\hline Billard's Butte & Boucher & Butte Colony \\
\hline Birch Pas Trail & Boucher Island & Butte Creek \\
\hline Bisson Island & Boucher Lake & Butte de la Croix \\
\hline Blais Island & Boueuse, Rivière & Butte du cheval Caille \\
\hline Blais Lake & Bouillon S.D. N 3067 & Butte du Paradis \\
\hline Blais Lake & Boule Creek & Butte S.D. N 4514 \\
\hline Blanc, Lac & Boule Creek S.D. N 3314 & Butte St. Pierre \\
\hline Blanchard Island & Bouleau, Décharge du & Butte St. Pierre S.D. $N^{\circ}$ \\
\hline Blanchard Lake & Bouleau, Décharge du & 5223 \\
\hline Blanche Lake & Bouleau, Demi-charge du & Buttes rondes, Prairie des \\
\hline Blanche, Rivière & Bouleau, Détroit du & Buttes rondes, Rivière des \\
\hline Blanche, Rivière & Bouleau, île au & Buttes, Lac des \\
\hline Blanche, Rivière & Bouleau, Île au & Byers Ferry \\
\hline Blancs, Lacs & Bouleau, Lac & \\
\hline Blatz-Nolin & Bouleau, Portage de & \\
\hline Blondeau Bay & Bouleau, Portage de & \\
\hline Blondeau Creek & Bouleau, Tête de & \\
\hline Blondeau Island & Bouleaux, Buttes aux & \\
\hline Blondeau Lake & Bouleaux, îles aux & \\
\hline Blondeau Mine & Bouquet de Saules & \\
\hline Blondeau River & Bourassa & \\
\hline Blondeau's House & Bourassa Creek & \\
\hline Blondeau's Point & Bourassa Lake & \\
\hline Boeuf, Détroit du & Bourassa S.D. N 1869 & \\
\hline Boeufs, Lac des & Bourbeuse, Rivière & \\
\hline Boire Lake & Bourbon, Rivière & \\
\hline Bois d'Orignal & Bourgeau Peninsula & \\
\hline Bois Fort, Butte & Bourgeault Lake & \\
\hline Bois Fort, Plaine du & Bourgeault Rapids & \\
\hline Bois, Lac du & Bourget Lake & \\
\hline
\end{tabular}

